\title{
GARCÍA MÁRQUEZ Y GARDEAZÁBAL ANTE CIEN A NOS DE SOLEDAD: UN DESAFIOO A LA INTERPRETACIÓN CRÍTICA
}

\author{
Por \\ RAYMOND L. WILLIAMS \\ Washington University
}

La narrativa colombiana de los últimos trece años ha sido dominada por la presencia de dos nombres: Gabriel García Márquez, por razones obvias, y más recientemente Gustavo Álvarez Gardeazábal, representante de la joven generación. De hecho, cuando el boom llegó a Colombia con Cien años de soledad (ya posterior a su éxito en América Latina), la Atenas Sudamericana se estremeció en shock. ${ }^{\prime}$ Pues el autor de una serie de novelas hasta entonces casi desconocidas fuera de limitados círculos intelectuales sacudió el país, tan tradicional, de su todavía vibrante enamoramiento de Isaacs, Rivera y Vargas Vila. Cien años de soledad fue, en efecto, a la vez un gran producto literario y todo un fenómeno en sí. El presente estudio considerará algunos de los efectos posteriores a lo que se podría denominar el "fenómeno Macondo" en Colombia.

El así llamado "fenómeno Macondo" fue ante todo una manifestación literaria: al crear las olas de significación nacional y universal que se dispersaban de Macondo, al captar y reescribir la historia de Colombia y América Latina, y al encarnar este contenido en un tono expertamente manejado, García Márquez captó la imaginación de los lectores de su patria. El fenómeno estuvo íntegramente ligado con la crítica: los colombianos jamás

1 Cien años de soledad llegó a la escena literaria en Colombia rela tivamente tarde en el año 1967. La primera noticia pública de la novela en Colombia a pareció en El Tiempo, el diario principal del país, en Julio de 1967. La siguiente cita es de la sección "Noticia de los libros" de El Tiempo (junto con una foto de la solapa de Cien años de soledad):

Solamente "por el forro" vamos a conocer los colombianos Cien años de soledad, "La primera verdadera novela de Gabriel García Márquez" según Hersán. Porque algún amigo trajo únicamente-y de contrabando- dos o tres ejemplares de México, y de uno de ellos reproducimos esta carátula, y el capítulo que aparece en nuestro suplemento de "Lecturas Dominicales". Las trabas de la aduana, y otras trabas, parece que demorarán demasiado la llegada a nuestras librerías de esta obra del glorioso Gabo, que tanta expectativa ha despertado. Por lo menos la primera edición-que, como se sabe, es de Sudamericana, de la Argentina - parece que ya está agotada...

El Tiempo [Bogotá], 16 de julio de 1967. 
habían visto tanta crítica sobre un autor vivo. Ya a principios de la década de los setenta los colombianos pudieron comentar la crítica misma, una situación algo curiosa en un país con una tradición bastante parca en lo que se refiere a la crítica literaria moderna. En los Estados Unidos el fenómeno se manifestó en un porcentaje relativamente alto de tesis doctorales sobre García Márquez, y en el mundo hispánico el autor que tal vez más impacto tiene a nivel popular, Mario Vargas Llosa, publicó su clásico estudio, Historia de un deicidio. ${ }^{2}$ En Colombia los suplementos dominicales, los órganos culturales mas influyentes, se encontraban sobre-cargados con reportajes y entrevistas con el ya héroe quizás anti-héroe nacional. Luego se produjeron varios efectos literarios o cuasi-literarios. Primero, se acabaron los premios literarios que existían antes de $1967 .^{3}$ La novela colombiana sufre, por un lado, de la imitación flagrante de Cien años de soledad, y por el otro, de la inevitable comparación con la obra maestra de Macondo con el resultado de que las demás novelas a parecieran siempre algo flojas. ${ }^{4} \mathrm{~A}$ principios y a mediados de la década de los setenta los jóvenes esritores ya hablaban de la necesidad de la superación, si no de la destrucción del así designado "mito" de García Márquez. De hecho, Álvarez Gardeazábal ha declarado públicamente y en artículos la necesidad de la superacion del mito de García Márquez. ${ }^{5} \mathrm{El}$ más joven Aguilera Garramuño, autor de una novela de bastante éxito en la segunda mitad de la década, publicó un artículo en 1974, antes de la aparición de su novela, que se refería a la misma necesidad que sentían los jóvenes narradores colombianos. ${ }^{6}$ Con este fondo-y repito que consta tanto de manifestaciones literarias como cuasi-literarias-nos acercamos al problema de la reacción de García Márquez y Ãlvarez Gardeazábal ante el monstruo

\footnotetext{
${ }^{2}$ Luis Pérez Botero analiza la crítica internacional ante García Márquez en "Garcia Márquez en la crítica internacional”, Revista Javeriana, 78, No. 388 (1972). Nota que de las 526 tesis de grado aceptadas (o en preparación) en las universidades de los Estados Unidos y en Canadá, doce versaban sobre García Márquez. Pérez nota además que "Este es un número de consideración, sobre todo si se tiene en cuenta que autores como Cervantes apenas atrajeron la atención a cinco estudiantes graduad os."

${ }^{3}$ El Premio Esso, el más importante en la narrativa, sobrevivió solamente dos años después de la publicación de Cien años de soledad.

${ }^{4}$ Las novelas de Flor Romero de Nohra y Germán Espinosa publicadas en esta época son evidentemente derivadas de Cien años de soledad.

5 Ver "Interview with Álvarez Gardeazábal" por Raymond L. Williams, Hispania, 59, No. 2 (mayo de 1976), 359-361.

6 Aguilera Garramuño publicó en 1975 su primera novela, Breve historia de todas las cosas (Buenos Aires: Ediciones de la Flor). El artículo fue "El mito de García Márquez", Arco, 163 (agosto de 1974), 29-35.
} 
que fue Cien años de soledad. Hay cuatro obras claves: La increible y triste historia de la cándida Eréndira y de su abuela desalmada (1972), El bazar de los idiotas (1974), El otoño del patriarca (1975) y El titiritero (1977).

Los cuentos del volumen La increible y triste historia fueron escritos entre 1968 y 1972 (con la excepción de uno, que aquí no figura) y por consiguiente son claves para el entendimiento del proceso de la narrativa colombiana posterior a Cien años de soledad. La estructura del volumen total, que consta de siete cuentos, es significante. Los seis primeros cuentos son breves, seguidos por el cuento titular de más de sesenta páginas. El primer cuento, "Un señor muy viejo con unas alas enormes", es absolutamente esencial para la experiencia total del volumen. Recordemos que una parte sustantiva del fenómeno Macondo ha sido el desparramo casi epidémico de interpretaciones acerca de Cien años de soledad: desde el ritmo de las frases hasta las connotaciones bíblicas, arquetípicas y semiológicas; figura entre las novelas más interpretadas en la historia de la literatura hispanoamericana. El primer cuento del volumen versa precisamente sobre este problema de la interpretación. ${ }^{7}$ Comienza con el descubrimiento por parte de un hombre sencillo, Pelayo, de un cuerpo humano algo extraño, en su patio: "Tuvo que acercarse mucho para descubrir que era un hombre viejo, que estaba tumbado boca abajo en el lodazal, y a pesar de sus grandes esfuerzos no podía levantarse, porque se lo impedían sus enormes alas". ${ }^{8}$ Esta frase rompe el desarrollo de un cuento dentro de un mundo ficticio hasta entonces comparable al nuestro; la presencia de un elemento de este tipo es un desafío a la razón. Su presencia genera inmediatamente una serie de interpretaciones. Para ser más preciso, surgen seis interpretaciones. Al recuperarse del asombro inicial, Pelayo llega a la conclusión de que el desconocido es un "náufrago solitario". La base de esta conclusión es su "buena voz de navegante". El lector considera caprichosa dicha afirmación: nuestra razón lógica rechaza la posibilidad de aceptar la idea misma de una supuesta "voz navegante" y encontramos risible la idea de Pelayo. La segunda interpretación es de una vecina que todos consideran muy sabia. En las palabras del narrador, "sabía todas las cosas de la vida y la muerte". Lo humorístico de su interpretación es su modus operandi: "a ella le bastó una mirada para sacarlos del error". Entonces declara: "Es un ángel". El lector se ríe de la afirmación por el contraste entre lo absurdo de la interpretación y la seguridad con que ella la manifiesta. Las tres interpre-

\footnotetext{
${ }^{7}$ En una reseña publicada sobre la versión inglesa del volumen el crítico John Sturrock muestra un desconocimiento de la obra de García Márquez y del lugar de estos cuentos dentro de la trayectoria completa de su obra. Critica la falta de "contenido" en los cuentos, y es precisamente de este tipo de lectura "interpretativa" de que García Márquez se burla.

${ }^{8}$ Gabriel García Márquez, La increíble y triste historia de la cándida Eréndira y de su abuela desalmada (México: Editorial Hermes, 1972), p. 11. Todas las citas son de esta edición.
} 
taciones que aparecen luego son todas de habitantes (ingenuos) del pueblo: proponen que puede ser "el alcalde del mundo", "un general de cinco estrellas" o "una estirpe de hombres alad os y sabios que se hicieron cargo del universo". El padre Gonzaga ofrece la sexta contribución al concluir que no es un ángel. Escribe cartas para que la jerarquía eclesiástica decida. Es notable que el “interpretador oficial" del pueblo, el cura, es el único que se niega a ofrecer interpretaciones concretas. En el pueblo, sin embargo, se rechazan los consejos del cura, y se sigue tratando al desconocido como un ángel. El final del cuento es ambiguo: el hombre alado aparentemente se recupera lo suficiente para poder volar; Elisenda lo observa alejarse y el cuento termina así:

Siguió viéndolo hasta cuando acabó de cortar la cebolla, y siguió viéndolo hasta cuando ya no era posible que lo pudiera ver, porque entonces ya no era un estorbo en su vida, sino un punto imaginario en el horizonte del mar. (p. 20)

En primer lugar, este final no ofrece ninguna conclusión racional acerca del hombre enigmático. Si el lector se atreve a contribuir con una séptima y última conclusión (mítica, freudiana o sociológica, sean las que sean las preferencias del crítico), mediante su aporte participa en el proceso de los habitantes del pueblo. Asimismo se sugiere la posibilidad de que el hombre alado sea puramente imaginativo porque se funde en "un punto imaginario en el horizonte del mar". "Un señor muy viejo con unas alas enormes" ejerce al menos tres funciones como introducción al volumen $\mathrm{y}$, a su vez, como reacción ante el fenómeno Macondo. Primero, es una parodia del proceso interpretativo. Como tal García Márquez se burla de toda una maquinaria crítica que ha surgido a partir de la publicación de Cien años de soledad. Esta parodia funciona como una advertencia al lector: los que insisten en interpretaciones racionales para fenómenos netamente inexplicables (que abundarán en el volumen) funcionarán como los Pelayos y Elisendas. Segundo, en el cuento prolifera el contenido mágico y milagroso. En este sentido es una reacción ante Cien años de soledad: lo mágico en sí es tema paródico en la narrativa colombiana de los setenta, como se verá más claramente en el caso de Álvarez Gardeazábal. Tercero, García Márquez afirma en este primer cuento el derecho a la invención, un hecho que el final del cuento hace evidente. El derecho a la invención en Colombia ha tendido a tener menos importancia que la "veracidad" histórica. ${ }^{9}$

\footnotetext{
${ }^{9}$ Ver John S. Brushwood, The Spanish American Novel: A Twentieth Century Survey (Austin: University of Texas Press, 1975). Brushwood destaca esta reafirmación del derecho del autor a la invención como una característica fundamental de la nueva novela hispanoamericana. Señala que principia en los años cuarenta con la publicación de cuatro novelas claves: El señor Presidente (1946), Al filo del agua (1947), Adán Buenosayres (1948) y El reino de este mundo (1949).
} 
De los cinco cuentos restantes del volumen, tres versan sobre problemas relacionados con la interpretación y la imaginación, afirmando así lo sugerido en el primer cuento. Dos, "Muerte constante más allá del amor" y el cuento titular, apuntan más bien hacia El otoño del patriarca.

El problema interpretativo en "El ahogado más hermoso del mundo" es la identidad de un cadáver enorme que llega a las playas de un pueblo con unos habitantes igualmente sencillos como los del primer cuento. Otra vez se hace la interpretación sin ninguna base racional: deciden que es una persona llamada "Esteban porque "Tiene cara de llamarse Esteban" (p. 52). Esta interpretación predomina inmediatamente: "Era verdad. A la mayoría le bastó con mirarlo otra vez para comprender que no podía tener otro nombre". Con el pasar del tiempo se apropian de él, dándole una identidad como miembro del pueblo. Al final casi todos los habitantes hasta lo consideran pariente. El último paso en ese proceso interpretativo es el efecto que esta interpretación ejerce sobre el pueblo: una vez miembro de la comunidad, cambia radicalmente su visión de sí mismos y estimula sus poderes imaginativos: "tuvieron conciencia por primera vez de la desolación de sus calles, la aridez de sus patios, la estrechez de sus sueños, frente al esplendor y la hermosura de su ahogado" (p. 56). La culminación del cuento es una liberación del pueblo: el tema es la imaginación. La imaginación y los milagros también son el enfoque de "El último viaje del buque fantasma" y "Blacamán el bueno vendedor de milagros".

El tema del poder y su manipulación surge en "Muerte más allá del amor". El senador Onésimo Sánchez es el prototipo del general en El otoño del patriarca. El senador sufre el mismo terror de la muerte y la relación entre él y su amante es precisamente igual a la asexualidad de la relación niño-madre del general en la novela. Su poder político otra vez depende del manejo de lo visible. La figura exageradamente autoritaria que representa la abuela en el cuento titular también apunta hacia el tema del poder desarrollado más tarde en la novela del dictador. Este cuento sintetiza todos los elementos narrativos examinados hasta ahora: la interpretación, la imaginación y el poder. También es un resumen de los otros más concretos por el hecho de incluir los mismos personajes de esos cuentos: aparecen un hombre con alas, Onésimo Sánchez, Blacamán el bueno y la mujer convertida en araña, entre otros. Es la interpretación de los eventos desde el principio lo que le permite explotar y oprimir a la nieta desde las primeras páginas del texto. La abuela funciona como interpretadora por excelencia a lo largo del texto, y sus interpretaciones vis-a-vis la nieta son siempre opresivas. ${ }^{10}$

10 Ver el principio y el fin del cuento (pp. 98 y 149) y el papel interpretativo de la abuela en ellos. 
La próxima reacción notable ante Cien años de soledad y el fenómeno Macondo fue El bazar de los idiotas, la cuarta novela de Álvarez Gardeazábal. ${ }^{11}$ Es una novela paródica de Cien años de soledad en varios niveles. Un logro importante de la novela de García Márquez es su empleo de la exageración: es un mundo hiperbólico donde todo es posible porque se eliminan los límites normales de lo "real" y lo "normal". Ãlvarez Gardeazábal se vale igualmente de la exageración, pero da un paso más: se burla de ella, la parodia. El bazar de los idiotas no solamente contiene la exageración garciamarquezana, sino que versa sobre la misma. Es una novela sobre la exageración, y el narrador mismo comenta los procesos hiperbólicos que se ven. Por ejemplo, dice lo siguiente de los resultados exagerados que tuvo el onanismo entre la juventud:

Los curas en sus sermones, los obispos en sus pastorales, los médicos por la televisión, los padres de familia en los almuerzos, los directores de los colegios, los profesores, todos los que podían hablar, hicieron campaña para mermar la oleada. Los peligros de la masturbación fueron sacados a la luz. Se llegó a exagerar tanto que se dijo que la masturbación era muchísimo más peligrosa que la marihuana, que no hace daño a nadie. Sólo ahora, a meses de la noticia que Inesista dio con una ingenuidad asombrosa, la moda ha cesado un poco y los masturbantes ya no gritan tanto. (mi énfasis, p. 260)

Cuando los idiotas alcanzan fama nacional el narrador observa: "Los de la revista Siete Días no inventaron más mentiras porque verdaderamente no pudieron" (p. 228).

El fenómeno exagerado de la popularidad de Macondo es parte de la parodia de Álvarez Gardeazábal. En Cien años de soledad Macondo es un pueblo pequeño, aislado $y$, no nos olvidemos, inventado. A partir de la publicación de la novela (no en la obra misma) Macondo alcanza fama universal. Álvarez Gardeazábal toma este fenómeno extra-literario y se burla de él al crear una obra dentro de la cual un pueblo (un pueblo no inventado) experimenta la misma fama mundial que Macondo tuvo extraliterariamente. Las implicaciones burlescas son especialmente interesantes cuando se comparan estos fenómenos: mientras Macondo atrae fama mundial a causa del trabajo literario de García Márquez, Tuluá alcanza la misma fama dentro de la obra de Álvarez Gardeazábal a causa de la masturbacción de dos adolescentes. (En efecto, Álvarez Gardeazábal ha creado un paralelo entre la escritura de García Márquez y la masturbación de unos adolescentes.)

Por otra parte, la estructura y la manera de concebir El bazar de los idiotas es una parodia de Cien años de soledad. García Márquez crea una obra de estructura circular en que reescribe la historia. Como ya ha sido bien

1 El bazar de los idiotas (Bogotá: Plaza \& Janés, 1974). Todas las citas son de esta edición. 
documentado, García Márquez ha escrito una historia inventada pero a veces más históricamente válida que las historias "oficiales" de Colombia. La huelga bananera es un buen ejemplo de la veracidad increíble de partes de esta novela. Álvarez Gardeazábal, en cambio hace la parodia de la re-escritura de la historia "olvidada" de Tuluá. La "historia olvidada" es esencialmente una invención burlesca de dos idiotas que, si existieron (cosa poco probable), no tienen ninguna importancia en la historia de Colombia. Mientras García Márquez cierra su obra con un círculo perfecto, Álvarez Gardeazábal crea las mismas expectativas para, al final, sencillamente acabar con los idiotas algo Deus ex-machina.

Dos novelas posteriores, El otoño del patriarca de García Márquez y $E l$ titiritero de Álvarez Gardeazábal marcan el fin, si no la destrucción, del fenómeno Macondo y los procesos interpretativos que con él se asociaban. Con Elotoño del patriarca García Márquez mismo se libera de Macondo y sus reverberaciones universales. Su dictador tiene más resonancia a nivel latinoamericano que estrictamente colombiano o universal, lo cual representa un cambio evidente en su narrativa. Los personajes y otros elementos de la novela no ofrecen las connotaciones arquetípicas que tanto interpretaban los críticos del ciclo de Macondo. El enfoque de la novela es la personalidad del dictador. Como ha señalado Ángel Rama, el interés del novelista es la sicología del dictador latinoamericano. ${ }^{12}$ Después de la publicación de $L a$ increible y triste historia, tal como lo hemos comentado, estamos conscientes de la ingenuidad de atribuir características de la madre arquetípica a la madre o la mujer del General; ni la una ni la otra es una Úrsula.

El titiritero de Álvarez Gardeazábal, publicada dos años después de la novela del dictador, representa la culminación del proceso de ruptura y alejamiento del fenómeno Macondo; al mismo tiempo Álvarez Gardeazábal ataca directamente a los críticos interpretativos. La novela trata de unos acontecimientos que giran alrededor de la muerte de un estudiante en la Universidad del Valle en 1971. El presente temporal es el 26 de febrero de 1986, el decimoquinto aniversario del fallecimiento de éste, Édgar Mejía Vargas. En este presente Mejía Vargas desempeña el papel de héroe político. Una gran parte de la novela narra los hechos relacionados con su muerte y con los personajes en la universidad: el rector Ollano, una estudiante llamada Vicky que pierde la razón a causa de su experiencia universitaria. También figuran las narraciones de un profesor de la Universidad del Valle que se describe como creador de la obra que el lector tiene en sus manos.

12 Ver Ángel Rama, Los dictadores latinoamericanos (México: Fondo de Cultura Económica, 1976). 
La ausencia de Tuluá y de todo lo que había llegado a significar el mundo tulueño es la manifestación más evidente de la reacción en contra del fenómeno Macondo: se acaban el ciclo tulueño con su pequeño Macondo del Valle del Cauca, sus acontecimientos maravillosos y mágicos, y a la vez desaparecen los mismos personajes repetidos desde La tara del papa hasta $E l$ bazar de los idiotas. El titiritero, como El otoño del patriarca, es una novela menos ambiciosa-que pretende mucho menos precisamente por la temática tan limitada y local. El asunto central del conflicto universitario ni intenta alcanzar la universalidad de un Macondo o tal vez de un Tuluá. El profesor/narrador de esta novela expresa algunas de las tendencias desarrolladas por Álvarez Gardeazábal a lo largo de la década. Por ejemplo, una manifestación explícita de la reacción negativa ante la maquinaria crítica / comercial que emanaba de Cien años de soledad es la sugestión por parte del profesor/narrador acerca de las editoriales:

Como no soy la agencia de doña Carmen Balcells ni mucho menos la oficina publicitaria de Harper Row, tengo que ceñirme a la experiencia, a mis lecturas y a mi investigación, lenta, callada, pero de todas maneras investigacion... ${ }^{13}$

Pero tal vez aun más notable en el contexto de este estudio es su actitud ante la crítica. El profesor/narrador dice, por ejemplo, lo siguiente:

Claro que estoy partiendo de la base de que usted es un lector común, porque si fuera uno de esos minuciosos, depredadores, que se llaman críticos y que además de encontrar cómo formo las frases, sin verbos reflexivos, con exceso o ausencia de adjetivos, también buscan las varias posiciones del narrador y la manera cómo manejo el tiempo y la forma... me encontrará unos mitos que no he querido hacer, unos líos que nunca han pasado por mi mente y defenderá, como todos los críticos, su sistema ideológico diciéndome que mi novela es la obra de un reaccionario o de un pequeño burgués, que fue incapaz de darle suficiente calidad social al arte. (p. 118)

Luego afirma lo ya sugerido en sus comentarios: "yo le tengo un desprecio muy crecidito a los críticos"(p. 119). El profesor de la novela nunca explica en detalle precisamente por qué les tiene tanto desprecio a esos monstruos que se llaman "críticos", pero la actitud comunicada se semeja a lo propuesto por García Márquez en el volumen de cuentos ya discutido: se nota un cinismo y falta de confianza ante los procesos interpretativos y una falta de fe en la razón. Mientras más "minucioso" o científico sea el trabajo del crítico, más dudoso es para el novelista. Incluso ataca directamente la semiología en su novela, otra vez por medio de su profesor/narrador: "Para hacerlo [se refiere

\footnotetext{
${ }^{13}$ Gustavo Álvarez Gardeazábal, El titiritero (Bogotá: Plaza y Janés, 1977), p. 63. Todas las citas son de esta edición.
} 
a la creación de su novela] no he tenido que ponerme a montar la novela sobre bases epistemológicas o sobre discursos semióticos pasados de moda" (p. 227).

En fin, si dejamos al lado brevemente las obras literarias de los respectivos autores, y tomamos en cuenta sus propias afirmaciones en cuanto a los procesos críticos, también notamos su desafío a la interpretación crítica. En un artículo publicado recientemente por García Márquez, describe la naturaleza fundamentalmente fantástica de la realidad latinoamericana, negando así los esfuerzos de los interpretadores "racionalistas" de esa realidad. En este artículo García Márquez invierte, por ejemplo, la explicación racionalista del fenómeno de frijoles saltarines. Dice lo siguiente acerca de ellos:

Racionalistas benévolos me han explicado que su movilidad se debe a una larva viva que tienen dentro, pero la explicación me parece pobre: lo maravilloso no es que los frijoles se muevan porque tengan una larva dentro, sino que tengan una larva dentro para que puedan moverse. ${ }^{14}$

Incluso ha negado en numerosas ocasiones las interpretaciones que, lógicamente (dado el contenido increíble de su obra), se refieren a la "fantasía" de la misma. Concluye así en su artículo: "La realidad es mejor escritor que nosotros. Nuestro destino, y tal vez nuestra gloria, es tratar de imitarla con humildad, y lo mejor que nos sea posible". Tal afirmación niega una gran parte de la interpretación crítica de la obra de García Márquez según los sistemas racionales que empleamos.

Álvarez Gr rdeazábal ha sido aún más agresivo en lo que se refiere a las últimas tendencias críticas. En un artículo recientemente publicado, titulado apropiadamente "Los daños de la semiología", ataca vehementemente todas las vertientes de la crítica reciente-desde el estructuralismo en general, hasta el sicoanálisis, el marxismo y la semiología. ${ }^{15}$ Aunque bastante más específico, y también con tono más emocionado que lo notado en García Márquez, comparte con él una negación de todos los esfuerzos de la crítica reciente. Su sugerencia final de que "la literatura hay que enseñar a gustarla" es una generalización poco relacionada con las tendencias principales de la crítica contemporánea y, claro, una negación de las pocas aproximaciones críticas a su propia obra.

\footnotetext{
14 Gabriel García Márquez, "Fantasía y creación artística en América Latina y el Caribe", Texto Crítico, 14 (julio-septiembre de 1979), 3-8.
}

15 Gustavo Álvarez Gardeazábal, "Los daños de la semiologia”, El Café Literario, 12 (noviembrediciembre de 1979), 36-37. 
El proceso de la novela colombiana tal como se ve en sus dos representantes máximos muestra un desarrollo paradójico pero en una dirección clara desde la publicación de Cien años de soledad. El fenómeno Macondo, indudablemente lo mejor que ha visto la novela colombiana en lo que va del siglo, causó reacciones literarias tanto para García Márquez como para Álvarez Gardeazábal. Los dos novelizaban a base de un regionalismo, universalismo e irracionalismo como impulso original del fenómeno, pero aun mientras se han ido alejando de Macondo y Tuluá, respectivamente, han mantenido una actitud desafiadamente anti-racional ante su propia creación, una actitud que rechaza la interpretación crítica. 